\title{
Shorter telomere length of T-cells in peripheral blood of patients with lung cancer
}

This article was published in the following Dove Press journal:

OncoTargets and Therapy

4 May 2016

Number of times this article has been viewed

\author{
Yaqin Qian ${ }^{1-3, *}$ \\ Tingting Ding ${ }^{1-3, *}$ \\ Lijuan $\mathrm{Wei}^{2}$ \\ Shui $\mathrm{Cao}^{2-4}$ \\ Lili Yang ${ }^{1-3}$
}

'Department of Immunology, Tianjin Medical University Cancer Institute and Hospital, Tianjin Medical University, Tianjin, People's Republic of China; ${ }^{2}$ National Clinical Research Center of Cancer. Tianjin, People's Republic of China; ${ }^{3}$ Tianjin Key Laboratory of Cancer Immunology and Biotherapy, Tianjin, People's Republic of China; ${ }^{4}$ Department of Biotherapy, Tianjin Medical University Cancer Institute and Hospital, Tianjin Medical University, Tianjin, People's Republic of China

*These authors contributed equally to this work
Correspondence: Lili Yang

Department of Immunology, Tianjin Medical University Cancer Institute and Hospital, Tianjin Medical University, Huanhuxi Road, Tiyuanbei, Hexi District, Tianjin 300060, People's Republic of China

Tel +8622 23340123 Ext 6003

Fax +8622 23537796

Email yanglili@tjmuch.com
Purpose: Telomere shortening occurs in tumor tissues and peripheral blood lymphocytes of many common human malignancies, including lung cancer, but its variation in T-cells has never been investigated. Thus, the aim of this study was to assess telomere length in T-cells and its correlation with the clinical characteristics of patients with lung cancer.

Patients and methods: A total of 40 patients with lung cancer but without prior cancer history and 25 healthy individuals were selected. T-cells were isolated and their telomere lengths were measured using quantitative real-time polymerase chain reaction methods.

Results: Telomere length in T-cells was significantly shorter in patients with lung cancer than in controls $(P<0.001)$. Shorter telomere length was significantly associated with increased clinical stage $(P=0.008)$ and distant metastasis $(P=0.028)$. Naïve T-cells from patients with lung cancer had significantly decreased telomere length when compared with those from controls $(P=0.012)$.

Conclusion: The shortened telomere length in T-cells occurred in naïve T-cells and might be related to lung cancer progression.

Keywords: tumor, telomere's activity, naïve T-cell, immunocompromise, telomere shortening, T lymphocytes

\section{Introduction}

Lung cancer is one of the cancer types with the highest mortality rates worldwide because of its high invasion and rapid metastasis. ${ }^{1}$ The immune system plays an important role in controlling and eradicating lung cancer. One of the key factors is the T-cell-mediated antitumor effect. ${ }^{2,3} \mathrm{~T}$-cells significantly contribute to orchestrating effective antitumor immune responses by expressing an array of effector molecules. ${ }^{4,5}$ Nevertheless, multiple mechanisms of immune suppression may exist to suppress host immune responses, foster tumor growth, and help cancer cells evade immune surveillance. ${ }^{6} \mathrm{CD} 8^{+} \mathrm{T}$-cells in a lung tumor microenvironment are functionally impaired, ${ }^{7}$ and patients with lung cancer have an increased pool of regulatory T-cells with potent immunosuppressive features in the peripheral blood. ${ }^{8}$ Thus, we investigated the possible effect of telomere shortening on T-cell in the peripheral blood of patients with lung cancer to uncover important information on tumorigenesis and development.

Human telomeres are specialized structures consisting of a repetitive hexanucleotide (TTAGGG) region and an associated telomere-binding protein complex (shelterin) at the ends of linear chromosomes. ${ }^{9}$ The main function of telomeres is to protect chromosomes from end-to-end fusions, misrepair, and degradation. ${ }^{10,11}$ In human somatic cells, telomerase is not detected, and these cells lose telomeres progressively at a rate of 50-200 bp with each mitotic division. ${ }^{12,13}$ However, human lymphocytes are able to upregulate telomerase upon activation, thereby delaying telomere loss. ${ }^{14,15}$ 
Telomere length is an important factor affecting chromosomal stability and tumorigenesis. ${ }^{16}$ In the past decade, telomere length has emerged as a promising clinical marker for risk and prognosis prediction in patients with malignant disorders. ${ }^{17}$ In lung cancer, tumors have significantly shorter telomeres when compared with control tissues of non-smallcell lung cancer, ${ }^{18}$ and shorter telomere length correlates with worse overall survival and disease-free survival. ${ }^{19} \mathrm{Cells}$ of the immune system are under enormous proliferative demand; therefore, telomeric intactness is critical in preserving immune functions. ${ }^{20}$ However, environmental and external factors, including cancer, accelerate telomere loss in human lymphocytes. Lymphocytes in patients with lung cancer had shorter telomere lengths than those in controls. ${ }^{21}$ Telomere shortening in mononuclear cells in the peripheral blood may be a risk factor for lung cancer. ${ }^{21}$ However, few studies have examined telomere length in T-cells and its association with clinicopathological variables of lung cancer.

The aim of this study was to assess telomere length change in peripheral T-cells from patients with lung cancer to understand immune disorders in a tumor microenvironment.

\section{Patients and methods}

\section{Patients and healthy controls}

Patients with lung cancer $(\mathrm{n}=40)$ were selected from Tianjin Medical University Cancer Institute and Hospital (Tianjin, People's Republic of China) during 2013-2014. None of these patients had undergone tumor-related surgery, radiotherapy, chemotherapy, or other medical interventions before they participated in the study. Healthy controls $(n=25)$ were selected among healthy individuals who came to Tianjin Medical University Cancer Institute and Hospital for regular physical examination. The TNM (tumor, node, and metastasis) and clinical stages of the patients were based on the Union for International Cancer Control classification. The characteristics of the patients with lung cancer and healthy controls are shown in Table 1. All study procedures were approved by the Tianjin Cancer Institute and Hospital Ethics Committee. Written informed consent was obtained from all individual participants included in the study.

\section{Isolation of T-cells from peripheral blood}

Exactly $4 \mathrm{~mL}$ of peripheral venous blood was collected in a sodium heparin tube. T-cells were isolated using Human T-cell Enrichment Cocktail (RosetteSep ${ }^{\mathrm{TM}}$; StemCell Technologies, Inc., Vancouver, BC, Canada) in accordance with the manufacturer's instructions. The number of T-cells isolated from a $4 \mathrm{~mL}$ peripheral blood sample was $\sim 2 \times 10^{6}$.
Table I Characteristics of patients with lung cancer and controls

\begin{tabular}{lll}
\hline Patients/controls & Values & $P$-value \\
\hline Age, mean age (range) & & \\
Patients $(n=40)$ & $59(45-77)$ & \\
Stage I & $61(47-72)$ & \\
Stage II & $57(55-63)$ & \\
Stage III & $58(45-70)$ & \\
Stage IV & $61(50-77)$ & 0.318 \\
Controls $(n=25)$ & $55(45-64)$ & \\
Sex, male/female & & \\
Patients $(n=40)$ & $23 / 17$ & 0.39 \\
Controls $(n=25)$ & $12 / 13$ & \\
Smoke, yes $/$ no & & \\
Patients $(n=40)$ & $20 / 20$ & \\
Controls $(n=25)$ & $14 / 11$ & \\
\hline
\end{tabular}

The purity of T-cells was $<97 \%$, which was estimated by flow cytometry using a fluorescein-isothiocyanate-conjugated anti-CD3 antibody (BD Biosciences, San Jose, CA, USA).

\section{Naïve and memory cell purification}

$\mathrm{T}$-cell subsets were isolated from purified $\mathrm{CD}^{+} \mathrm{T}$-cells with PE-CD45RO and APC-CD45RA antibody (BD Biosciences) by the Aria flow sorter (BD Biosciences). Separation was performed in accordance with the manufacturer's protocol. The subset purity was $>95 \%$ in the post-sort analyses. Naïve (CD45RA+CD45RO-) and memory (CD45RO+CD45RA-) $\mathrm{T}$-cells were separated and placed in different tubes.

\section{Measurement of telomere length}

DNA was extracted from T-cells by using the PureLink Genomic DNA Kits (Tiangen, Beijing, People's Republic of China) in accordance with the manufacturer's instructions, and relative telomere lengths were measured following a modified version of the quantitative real-time polymerase chain reaction (PCR)-based telomere assay. ${ }^{22}$ In brief, the telomere repeat copy number to single gene copy number $(\mathrm{T} / \mathrm{S})$ ratio was determined using the 7,500 real-time PCR system (Thermo Fisher Scientific's Waltham, MA, USA) in a 96-well format. Genomic DNA (10 ng) was used for either the telomere or hemoglobin ( $\mathrm{Hgb})$ PCR reaction. All samples were analyzed by both the telomere and $\mathrm{Hgb}$ reactions, and the analysis was performed in triplicate on the same plate. Each 96-well plate contained a five-point standard curve from 0.08 to $50 \mathrm{ng}$ using genomic DNA. The T/S ratio $\left(-\Delta C_{t}\right)$ for each sample was calculated by subtracting the median $\mathrm{Hgb}$ threshold cycle $\left(C_{\mathrm{t}}\right)$ value from the median telomere $C_{\mathrm{t}}$ value. The relative $\mathrm{T} / \mathrm{S}$ ratio $\left(-\Delta \Delta C_{\mathrm{t}}\right)$ was determined by subtracting the $\mathrm{T} / \mathrm{S}$ ratio of the $10.0 \mathrm{ng}$ standard curve point from the $\mathrm{T} / \mathrm{S}$ ratio of each 
unknown sample. The primers used were as follows: Tel-1 primer(CGG TTT GTT TGG GTT TGG GTT TGG GTT TGG GTT TGG GTT), Tel-2 primer (GGC TTG CCT TAC CCT TAC CCT TAC CCT TAC CCT TAC CCT), Hgb-1 primer (GCT TCT GAC ACA ACT GTG TTC ACT AGC), and Hgb-2 primer (CAC CAA CTT CAT CCA CGT TCA CC).

\section{Statistical analysis}

Telomere length was assessed by the Wilcoxon rank sum test between cases with lung cancer and controls. The correlation between age and relative telomere length was determined using the Spearman correlation coefficient. Telomere length was analyzed as a continuous variable and as a categorical variable. Telomere length was dichotomized at the median value of patients included in the comparison by clinicopathological factors, including sex, age, smoking history (never smoker and ever smoker), lymph node dissemination, and distant metastasis. The categorized telomere length by clinicopathological factors were compared using the chi-squared test. All statistical analyses were performed using SPSS 20.0 software package (IBM Corporation, Armonk, NY, USA); statistical significance was considered at $P$-value $<0.05$.

\section{Results}

\section{Clinical characteristics of patients with lung cancer and controls}

The characteristics of the 40 patients with lung cancer and 25 controls are summarized in Table 1 . The mean age of the patients with lung cancer was $59 \pm 7$ years (range: $45-77$ ) and that of the controls was $55 \pm 6$ years (range: $45-64 ; P=0.27$ ). All the subgroups and the controls were matched in age $(P=0.745)$. Among the 40 cases and 25 controls, 23 (58\%) and $12(48 \%)$ were male, respectively $(P=0.39)$. Among the participants, 20 patients and 14 controls had a history of smoking $(P=0.30)$.

\section{Shorter telomere length in T-cells from patients with lung cancer}

A comparison of telomere lengths in peripheral blood T-cells between patients with lung cancer and controls is shown in Figure 1A. The telomere length in T-cells from the patients with lung cancer was significantly shorter $(n=40$, median $=2.089,95 \%$ confidence interval $[\mathrm{CI}], 1.858-3.143$ ) than that in T-cells from the controls $(\mathrm{n}=25$, median $=4.449$, 95\% CI, 4.136-5.812; $P<0.001)$ after adjusting for age and sex. In the healthy controls, the telomere length in T-cells declined progressively with increased age $(r=-0.574$, $P=0.002$; Figure 1B). Although a similar trend was observed in patients with lung cancer, the correlation between telomere length and age was not statistically significant $(r=-0.018$, $P=0.910$; Figure 1B).

\section{Shorter telomere length in T-cells from patients with advanced lung cancer}

To compare further the telomere lengths between healthy controls and patients with lung cancer, we divided the patients
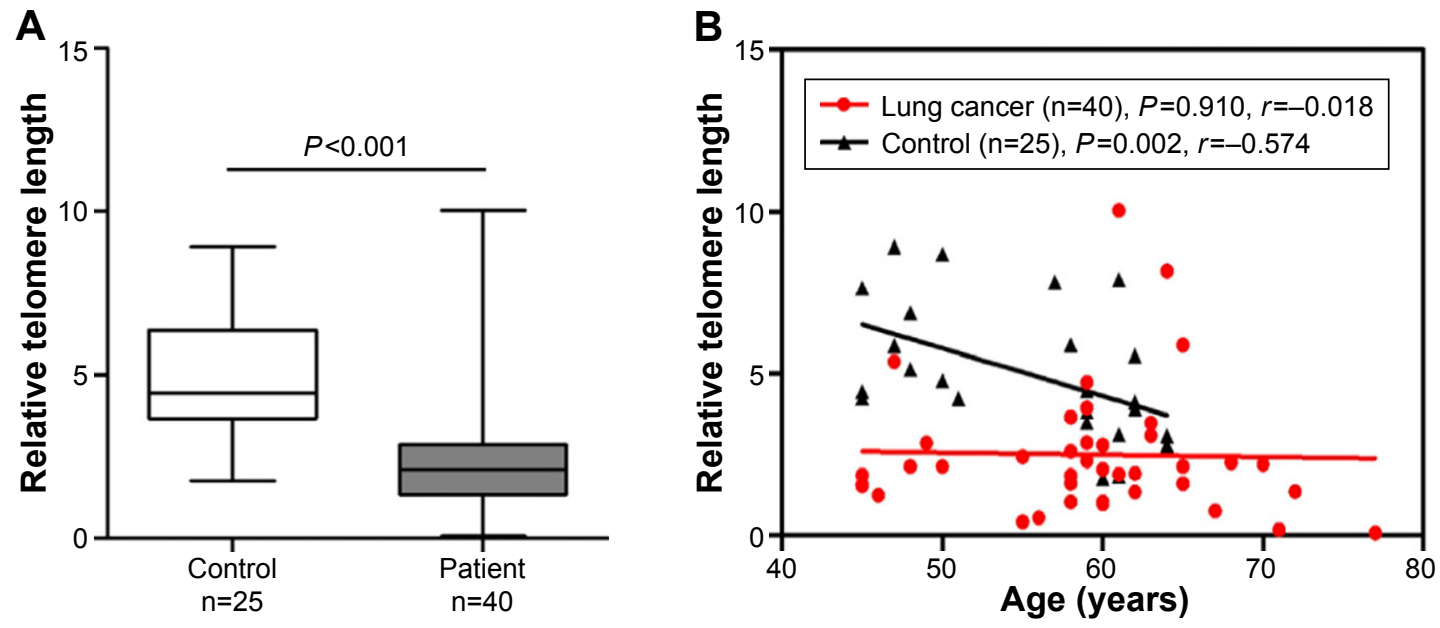

Figure I Shorter telomere length in T-cells from patients with lung cancer.

Notes: The telomere lengths in T-cells from patients with lung cancer and healthy controls were compared using the Wilcoxon rank sum test. The correlation between telomere length and age was assessed using the Spearman correlation coefficient. $P<0.05$ was considered statistically significant (A) The telomere length in T-cells from the patients with lung cancer was significantly shorter than that in T-cells from the controls $(P<0.00 I)$. (B) The telomere length ( $y$-axis) in T-cells relative to $H g b$ was inversely correlated with the age in the controls $(r=-0.574, P=0.002)$. Although a similar trend was observed in patients with lung cancer, the correlation was not statistically significant $(r=-0.018, P=0.910)$.

Abbreviation: Hgb, hemoglobin. 
into early-stage group (stages I and II) and advanced-stage group (stages III and IV). T-cells in both groups had significantly shorter telomere lengths than those in the controls (early stage $[$ median $=2.830,95 \%$ CI, $1.957-4.578]$ vs control [median $=4.449,95 \%$ CI, 4.136-5.812, $P=0.016]$; advanced stage $[$ median $=1.865,95 \% \mathrm{CI}, 1.375-2.800]$ vs control [median $=4.449,95 \% \mathrm{CI}, 4.136-5.812, P<0.001])$. A comparison of the early- and advanced-stage patients showed that the latter had a significantly shorter telomere length than the former $($ median $=2.830,95 \%$ CI, $1.957-4.578$ vs median $=1.865$, 95\% CI, 1.375-2.800, $P=0.008$; Figure 2A).

A previous study suggested that telomere length varies according to tumor histology; ${ }^{21}$ accordingly, we also performed an analysis between patients with different histological types of lung cancer and the controls. The telomere lengths in patients with adenocarcinoma (AC) and squamous cell carcinoma (SCC) were both shorter than those in the controls (AC [median $=2,195,95 \%$ CI, 1.824-3.427] vs controls $[$ median $=4.449,95 \% \mathrm{CI}, 4.136-5.812, P<0.001]$; SCC $[$ median $=1.955,95 \%$ CI, 1.262-3.026] vs controls [median $=4.449,95 \%$ CI, 4.136-5.812, $P=0.001$ ]; Figure 2B). However, we did not find a significant difference between the patients with $\mathrm{AC}$ and $\mathrm{SCC}(P=0.407$; Figure 2B).

\section{Relationship between clinicopathological parameters and telomere length in patients with lung cancer}

In consideration of the potential influence of telomere length on the T-cell antitumor effect, telomere length may be associated with the parameters of tumor development and metastasis. To test this hypothesis, we analyzed the relationship between clinicopathological parameters and telomere length in patients with lung cancer. Telomere length was significantly correlated with distant metastasis (metastasis-present patients [median $=0.875,95 \% \mathrm{CI}$, $0.507-1.819$ ] vs metastasis-absent patients [median $=2.225$, 95\% CI, 2.168-3.725], $P=0.028$ ). However, telomere length showed no significant correlations with other clinicopathological characteristics, including sex, age, smoking history, and lymph node metastasis (Table 2).

\section{Short telomere length in naïve T-cells from patients with lung cancer}

The telomere lengths in the purified naïve and memory T-cells from patients with lung cancer $(n=7)$ and healthy controls $(n=4)$ were measured to explore whether telomere dysfunction is a feature of antigen exposure (Figure 3 ). The naïve T-cells from patients with lung cancer had significant shorter telomere length than those from the healthy controls ( median $=1.166,95 \%$ CI, 0.812-1.779 vs median $=2.942$, $95 \% \mathrm{CI}, 1.400-4.814, P=0.012$ ), whereas no difference was observed in the memory T-cells between the patients with lung cancer and the controls (median $=1.420,95 \%$ CI, 0.739 2.533 vs median $=1.302,95 \%$ CI, 0.991-1.603, $P=0.927$; Figure 3 ). We also compared the difference between naïve T-cells and memory T-cells from patients with lung cancer and the controls, respectively. As expected, the telomere length in the naïve T-cells was significantly longer than that in the memory cells from the controls (median $=2.942,95 \%$ CI, $1.400-4.814$ vs median $=1.302,95 \%$ CI, 0.991-1.603, $P=0.028)$, whereas no significant difference was observed in patients with lung cancer $(P=0.805$; Figure 3$)$.
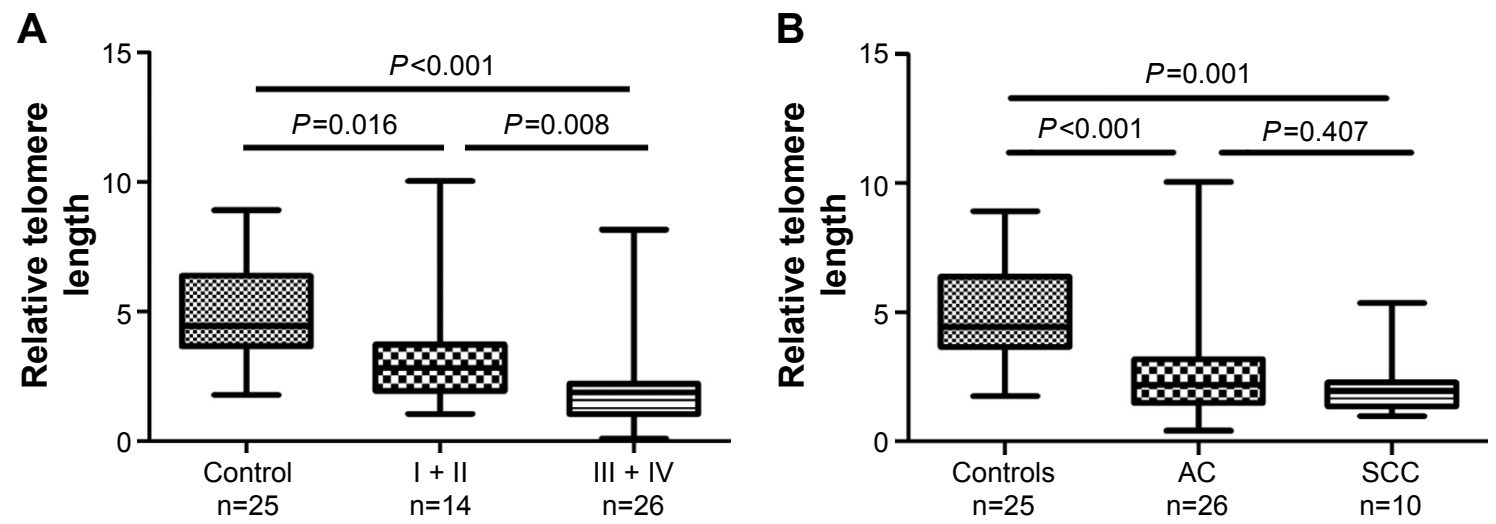

Figure 2 Telomere length at different clinical stages compared with controls.

Notes: The telomere lengths in T-cells from patients with lung cancer and healthy controls were compared using the Wilcoxon signed-rank test. (A) The patients were divided into early-stage group (stages I and II) and advanced-stage group (stages III and IV). The early- $(P=0.0 \mathrm{I} 6)$ and advanced-stage $(P<0.00 \mathrm{I})$ patients had T-cells with significantly shorter telomere lengths than the controls. (B) The telomere lengths in the patients with lung AC $(P<0.00 \mathrm{I})$ and SCC $(P=0.00 \mathrm{I})$ were both shorter than those in the controls; however, the difference between the two patient groups was not statistically significant $(P=0.407)$. All tests were two-sided; $P<0.05$ was considered statistically significant.

Abbreviations: AC, adenocarcinoma; SCC, squamous cell carcinoma. 
Table 2 Clinical characteristics and correlation analysis in patients with lung cancer

\begin{tabular}{|c|c|c|c|c|c|c|}
\hline \multicolumn{3}{|l|}{$n=40$} & \multicolumn{3}{|l|}{ Telomere length } & \multirow[t]{2}{*}{$P$-value } \\
\hline Characteristic & $\mathbf{n}$ & $\%$ & Median $(95 \% \mathrm{CI})$ & $\begin{array}{l}\text { Short telomere, } \\
\text { n (\%) }\end{array}$ & $\begin{array}{l}\text { Long telomere, } \\
\text { n (\%) }\end{array}$ & \\
\hline \multicolumn{7}{|l|}{ Sex } \\
\hline Male & 23 & 58 & $2.050(1.7 \mid 4-3.827)$ & $12(52)$ & II (48) & \\
\hline Female & 17 & 42 & $2.130(1.633-2.756)$ & $8(47)$ & $9(53)$ & 0.749 \\
\hline \multicolumn{7}{|l|}{ Age (years) } \\
\hline$\leq 60$ & 24 & 60 & $2.090(1.764-2.788)$ & $12(50)$ & $12(50)$ & \\
\hline$>60$ & 16 & 40 & $2.025(1.490-4.435)$ & $8(50)$ & $8(50)$ & 1.000 \\
\hline \multicolumn{7}{|l|}{ Smoke } \\
\hline Yes & 20 & 50 & $1.985(1.579-3.636)$ & II (55) & $9(45)$ & \\
\hline No & 20 & 50 & $2.130(1.526-3.262)$ & $9(45)$ & II (55) & 0.527 \\
\hline \multicolumn{7}{|c|}{ Lymph node metastasis } \\
\hline Present & 28 & 70 & $1.910(1.630-2.952)$ & $4(33)$ & $8(67)$ & \\
\hline Absent & 12 & 30 & $2.585(1.333-4.674)$ & $16(57)$ & $12(43)$ & 0.168 \\
\hline \multicolumn{7}{|l|}{ Distant metastasis } \\
\hline Present & 10 & 25 & $0.875(0.507-1.819)$ & $12(60)$ & $8(40)$ & \\
\hline Absent & 30 & 75 & $2.225(2.168-3.725)$ & $8(80)$ & $2(20)$ & 0.028 \\
\hline Total & 40 & 100 & & & & \\
\hline
\end{tabular}

Abbreviation: $\mathrm{Cl}$, confidence interval.

\section{Discussion}

This study investigated the telomere length in T-cells from patients with lung cancer. We found a shorter telomere length in patients with lung cancer than in the controls. Furthermore, telomere length was correlated with distant metastasis and increased clinical stage. Telomere shortening occurred in the naïve T-cells but not in the memory T-cells. These findings

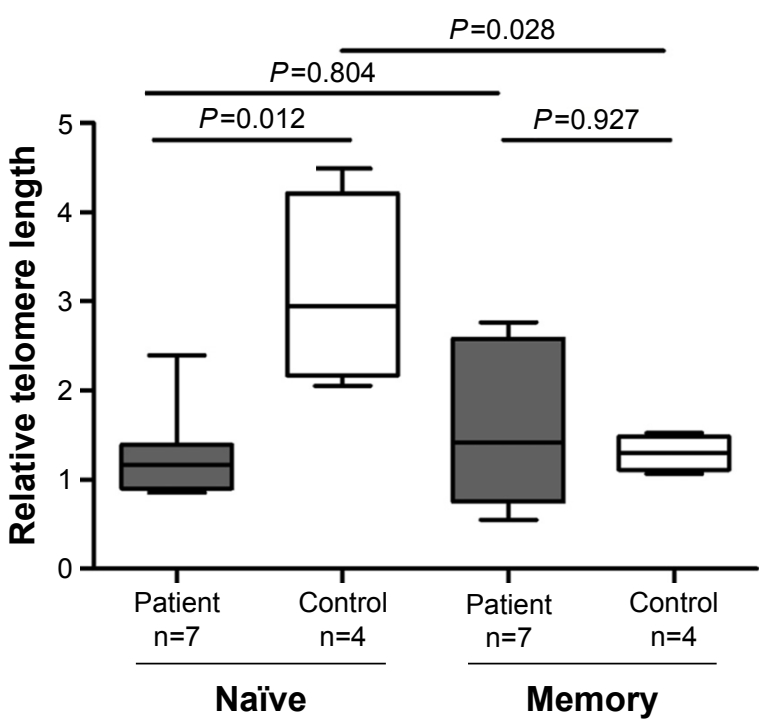

Figure 3 Telomere length in naïve T-cells from patients with lung cancer. Notes: The telomere lengths in purified naïve and memory T-cells from subsets of cases with lung cancer $(n=7)$ and healthy controls $(n=4)$ were compared using the Wilcoxon sum rank test. The mean relative telomere length in naïve T-cells was significantly longer than that in memory cells from the controls $(P=0.028)$, whereas no difference was observed in the cases $(P=0.804)$. The telomere length in naïve cells was significantly decreased in the cases compared with the controls $(P=0.0 \mid 2)$, whereas no difference was observed in memory cells $(P=0.927)$. suggested that the telomere length in peripheral T-cells, specifically naïve T-cells, is a potential diagnosis and prognostic marker for patients with lung cancer.

Telomere dysfunction has been found in many cancer types. Previous studies on breast cancer, ${ }^{23}$ lung cancer, ${ }^{24}$ and renal cancer ${ }^{25}$ showed that telomere length is significantly shorter in cancer tissues than in corresponding normal tissues. The telomere length of peripheral blood leukocytes was also frequently examined and associated with cancer risk for various malignancies; however, the conclusions were inconsistent. ${ }^{21}$ Considering that telomere length may vary across major blood cell types ${ }^{26}$ this study focused on purified T-cells, which are highly dependent on telomere repair because of the high rate of turnover. ${ }^{22}$ As expected, shorter telomeres were found in patients with lung cancer than in the healthy controls, indicating that telomere loss influenced tumorigenesis. Moreover, the difference in telomere length between the patients and the controls suggested the potential diagnostic value of telomere measurement in T-cells from patients with lung cancer.

In breast cancer, telomere shortening is reportedly more significant in the higher grades of cancer samples. ${ }^{23}$ In nonsmall-cell lung cancer, telomere attrition is associated with a poor clinical outcome. ${ }^{24}$ Consistent with this finding, the current results suggest that telomere shortening in T-cells is more pronounced in patients with distant metastasis and advanced stages than in non-metastasis and early stages. Therefore, shorter telomere length might be a reflection of clinical evolution, showing promise as a prognostic parameter. Previous studies reported that psychological 
stress,${ }^{27}$ body mass index,${ }^{28}$ physical activity, ${ }^{29}$ alcohol consumption, ${ }^{30}$ nutrition, ${ }^{31}$ and hormone ${ }^{32}$ may be associated with telomere length, thus the limitation of our study is that we did not consider the differences in these factors between patient and healthy control groups.

Telomere dysfunction in T-cells may also provide therapeutic clues. The competence of the immune system strictly depends on the renewal and clonal expansion of immune cells, especially T-cell and B-cell populations. ${ }^{33}$ Although tumor cells that express tumor-associated antigens can be recognized and attacked by T-cells, the antitumor effect of T-cells is often compromised to favor the evasion from the host's defenses by a number of mechanisms, including induction of the secretion of immunosuppressive cytokines, apoptosis of T-cells, and modulation of immune polarization. ${ }^{34}$ Wang et a ${ }^{35}$ suggested that lung cancer cell reduced IFN $\gamma$ expression in $\mathrm{CD}^{+} \mathrm{T}$-cells, which may serve as an important mechanism of tumor-induced immunosuppression. Montes et $\mathrm{al}^{36}$ observed that human T-cells from healthy donors incubated with tumor cells undergo a senescence-like phenotype, and both $\mathrm{CD} 8^{+}$and $\mathrm{CD} 4^{+}$senescent $\mathrm{T}$-lymphocytes suppressed the proliferation of normal T-cells. Mittal et al ${ }^{37}$ showed that murine lung cancer induced T-cell exhaustion by increasing the expression of inhibitory molecules and inducing the apoptosis of $\mathrm{CD}^{+} \mathrm{T}$-cells. The loss of telomere in T-cells possibly causes diminished T-cell function by inducing $\mathrm{T}$-cell senescence. Thus, monitoring telomere dynamics may reflect the status of the immune system and provide evidence for optimal biotherapy.

Studies have suggested that if telomeres shorten because of proliferative stress, then telomeres should be the shortest in memory cells that have been exposed to antigenic stimulation..$^{22}$ In the current study, naïve and memory populations were compared in patients with lung cancer and healthy controls to assess whether telomere dysfunction is a feature of antigen exposure. The telomere length in naïve cells was significantly decreased in the patients compared with that of controls, whereas no case-control difference was observed in the memory cells. Moreover, the telomere length in naïve T-cells from patients with lung cancer has no significant difference with respect to the memory T-cell population, which experienced proliferative stress. Thus, significant telomere loss mainly occurred in naïve T-cells. The telomere length in peripheral blood cells indirectly reflects the mitotic history of their precursors, hematopoietic stem cells. ${ }^{38}$ Evidence of myelodysplastic syndromes indicated that telomere attrition is acquired within the hematopoietic compartment. ${ }^{39}$ In our previous study, ${ }^{22}$ we also detected telomere attrition in T-cells. ${ }^{22}$ Thus, the assumptions that T-cells carry underlying mutations derived from their progenitor cells and short telomeres in naïve T-cells may point to an increased hematopoietic cell turnover, replicative senescence, and genetic instability during lung cancer development are tempting; however, future investigations should still be performed on hematopoietic stem cells and distinct cell populations.

Telomerase can compensate for telomere shortening, and telomerase activity is expressed at a low level in normal peripheral leukocytes..$^{40}$ However, the enzymatic activity of telomerase varies across different lymphocyte subsets. ${ }^{41,42}$ Furthermore, changes in telomerase activity vary across different T-cell subsets when exposed to cortisol. ${ }^{32}$ Whether telomere shortening in naïve T-cells instead of memory T-cells is due to immune cell-type-specific telomerase activity is still unknown, and clarifying this issue in further research is of interest.

A previous study provided evidence revealing that changes in the telomere length in T-cells are influenced by naïve T-cell percentage in total T-cells. ${ }^{43}$ However, our previous unpublished data show that the percentages of naïve T-cells between patients with lung cancer and age and sex-adjusted healthy controls are not significantly different, indicating a shorter telomere length in patients may not be ascribed to the reduction in naïve T-cell proportion in patients.

\section{Conclusion}

In summary, T-cells from patients with lung cancer possessed significantly shorter telomeres than those from healthy controls, and telomere length decreased with cancer progression. Telomere loss mainly occurred in the naïve T-cell population. Our findings provide novel insights into telomere dysfunction in a lung cancer microenvironment and contribute to the understanding of the proliferative capability of T-cells and the immune responses of patients. We intend to collect more samples to clarify our conclusion and explore further the underlying mechanism in our future study.

\section{Acknowledgments}

This work was supported by grants from the National Basic Research Program of China (973 program) (No 2012CB933304), Tianjin Natural Science Fund (14JCYBJC25500), and National Natural Science Foundation of China (81572265). We would like to thank Wei Sheng, MD, from the Immunology Program of the H Lee Moffitt Cancer Center in Tampa, FL, USA, for his technical assistance. 


\section{Disclosure}

The authors report no conflicts of interest in this work.

\section{References}

1. Lemjabbar-Alaoui H, Hassan OU, Yang YW, Buchanan P. Lung cancer: biology and treatment options. Biochim Biophys Acta. 2015;1856(2): $189-210$.

2. Freedman LR, Cerottini JC, Brunner KT. In vivo studies of the role of cytotoxic T cells in tumor allograft immunity. J Immunol. 1972;109(6): 1371-1378.

3. Hung K, Hayashi R, Lafond-Walker A, Lowenstein C, Pardoll D, Levitsky $\mathrm{H}$. The central role of CD4(+) T cells in the antitumor immune response. J Exp Med. 1998;188(12):2357-2368.

4. Andersson A, Yang SC, Huang M, et al. IL-7 promotes CXCR3 liganddependent T cell antitumor reactivity in lung cancer. J Immunol. 2009; 182(11):6951-6958

5. Weynants P, Wauters P, Coulie PG, Van den Eynde B, Symann M, Boon T. Cytolytic response of human $\mathrm{T}$ cells against allogeneic small cell lung carcinoma treated with interferon gamma. Cancer Immunol Immunother. 1988;27(3):228-232.

6. Karachaliou N, Cao MG, Teixido C, et al. Understanding the function and dysfunction of the immune system in lung cancer: the role of immune checkpoints. Cancer Biol Med. 2015;12(2):79-86.

7. Prado-Garcia H, Aguilar-Cazares D, Flores-Vergara H, Mandoki JJ, Lopez-Gonzalez JS. Effector, memory and naive CD8+ T cells in peripheral blood and pleural effusion from lung adenocarcinoma patients. Lung Cancer. 2005;47(3):361-371.

8. Erfani N, Mehrabadi SM, Ghayumi MA, et al. Increase of regulatory $T$ cells in metastatic stage and CTLA-4 over expression in lymphocytes of patients with non-small cell lung cancer (NSCLC). Lung Cancer. 2012;77(2):306-311.

9. Broccoli D. Function, replication and structure of the mammalian telomere. Cytotechnology. 2004;45(1-2):3-12.

10. McClintock B. The behavior in successive nuclear divisions of a chromosome broken at meiosis. Proc Natl Acad Sci U S A. 1939;25(8): 405-416.

11. de Lange T. How shelterin solves the telomere end-protection problem. Cold Spring Harb Symp Quant Biol. 2010;75:167-177.

12. Levy MZ, Allsopp RC, Futcher AB, Greider CW, Harley CB. Telomere end-replication problem and cell aging. J Mol Biol. 1992;225(4): 951-960.

13. Allsopp RC, Vaziri H, Patterson C, et al. Telomere length predicts replicative capacity of human fibroblasts. Proc Natl Acad Sci USA. 1992, 89(21):10114-10118.

14. Pan C, Xue BH, Ellis TM, Peace DJ, Diaz MO. Changes in telomerase activity and telomere length during human $\mathrm{T}$ lymphocyte senescence. Exp Cell Res. 1997;231(2):346-353.

15. Son NH, Joyce B, Hieatt A, Chrest FJ, Yanovski J, Weng NP. Stable telomere length and telomerase expression from naive to memory B-lymphocyte differentiation. Mech Ageing Dev. 2003;124(4):427-432.

16. Artandi SE, DePinho RA. Telomeres and telomerase in cancer Carcinogenesis. 2010;31(1):9-18.

17. Svenson U, Roos G. Telomere length as a biological marker in malignancy. Biochim Biophys Acta. 2009;1792(4):317-323.

18. Fernandez-Marcelo T, Gomez A, Pascua I, et al. Telomere length and telomerase activity in non-small cell lung cancer prognosis: clinical usefulness of a specific telomere status. J Exp Clin Cancer Res. 2015;34:78.

19. Jeon HS, Choi YY, Choi JE, et al. Telomere length of tumor tissues and survival in patients with early stage non-small cell lung cancer. Mol Carcinog. 2014;53(4):272-279.

20. Andrews NP, Fujii H, Goronzy JJ, Weyand CM. Telomeres and immunological diseases of aging. Gerontology. 2010;56(4):390-403.

21. Jang JS, Choi YY, Lee WK, et al. Telomere length and the risk of lung cancer. Cancer Sci. 2008;99(7):1385-1389.
22. Yang L, Mailloux A, Rollison DE, et al. Naive T-cells in myelodysplastic syndrome display intrinsic human telomerase reverse transcriptase (hTERT) deficiency. Leukemia. 2013;27(4):897-906.

23. Poonepalli A, Banerjee B, Ramnarayanan K, Palanisamy N, Putti TC, Hande MP. Telomere-mediated genomic instability and the clinicopathological parameters in breast cancer. Genes Chromosomes Cancer. 2008;47(12):1098-1109

24. Frias C, Garcia-Aranda C, De Juan C, et al. Telomere shortening is associated with poor prognosis and telomerase activity correlates with DNA repair impairment in non-small cell lung cancer. Lung Cancer. 2008;60(3):416-425.

25. Pal D, Sharma U, Khajuria R, Singh SK, Kakkar N, Prasad R. Augmented telomerase activity, reduced telomere length and the presence of alternative lengthening of telomere in renal cell carcinoma: plausible predictive and diagnostic markers. Gene. 2015;562(2):145-151.

26. Hoffmann J, Erben Y, Zeiher AM, Dimmeler S, Spyridopoulos I. Telomere length-heterogeneity among myeloid cells is a predictor for chronological ageing. Exp Gerontol. 2009;44(5):363-366.

27. Shalev I, Entringer S, Wadhwa PD, et al. Stress and telomere biology: a lifespan perspective. Psychoneuroendocrinology. 2013;38(9): $1835-1842$.

28. Rode L, Nordestgaard BG, Weischer M, Bojesen SE. Increased body mass index, elevated C-reactive protein, and short telomere length. J Clin Endocrinol Metab. 2014;99(9):E1671-E1675.

29. Cherkas LF, Hunkin JL, Kato BS, et al. The association between physical activity in leisure time and leukocyte telomere length. Arch Intern Med. 2008;168(2):154-158.

30. Pavanello S, Hoxha M, Dioni L, et al. Shortened telomeres in individuals with abuse in alcohol consumption. Int J Cancer. 2011;129(4): 983-992.

31. Paul L. Diet, nutrition and telomere length. J Nutr Biochem. 2011;22(10): 895-901.

32. Choi J, Fauce SR, Effros RB. Reduced telomerase activity in human T lymphocytes exposed to cortisol. Brain Behav Immun. 2008;22(4): 600-605.

33. Kaszubowska L. Telomere shortening and ageing of the immune system. J Physiol Pharmacol. 2008;59(suppl 9):169-186.

34. Whiteside TL. Immune responses to malignancies. J Allergy Clin Immunol. 2010;125(2 suppl 2):S272-S283.

35. Wang F, Xu J, Zhu Q, et al. Downregulation of IFNG in CD4(+) T cells in lung cancer through hypermethylation: a possible mechanism of tumor-induced immunosuppression. PLoS One. 2013;8(11):e79064.

36. Montes CL, Chapoval AI, Nelson J, et al. Tumor-induced senescent $\mathrm{T}$ cells with suppressor function: a potential form of tumor immune evasion. Cancer Res. 2008;68(3):870-879.

37. Mittal R, Chen CW, Lyons JD, et al. Murine lung cancer induces generalized T-cell exhaustion. J Surg Res. 2015;195(2):541-549.

38. Sakoff JA, De Waal E, Garg MB, et al. Telomere length in haemopoietic stem cells can be determined from that of mononuclear blood cells or whole blood. Leuk Lymphoma. 2002;43(10):2017-2020.

39. Lange K, Holm L, Vang Nielsen K, et al. Telomere shortening and chromosomal instability in myelodysplastic syndromes. Genes Chromosomes Cancer. 2010;49(3):260-269.

40. Weng NP, Hathcock KS, Hodes RJ. Regulation of telomere length and telomerase in $\mathrm{T}$ and $\mathrm{B}$ cells: a mechanism for maintaining replicative potential. Immunity. 1998;9(2):151-157.

41. Ouyang Q, Baerlocher G, Vulto I, Lansdorp PM. Telomere length in human natural killer cell subsets. Ann N Y Acad Sci. 2007;1106: $240-252$.

42. Weng NP, Granger L, Hodes RJ. Telomere lengthening and telomerase activation during human B cell differentiation. Proc Natl Acad Sci U S A. 1997;94(20):10827-10832.

43. Lin Y, Damjanovic A, Metter EJ, et al. Age-associated telomere attrition of lymphocytes in vivo is co-ordinated with changes in telomerase activity, composition of lymphocyte subsets and health conditions. Clin Sci (Lond). 2015;128(6):367-377. 


\section{Publish your work in this journal}

OncoTargets and Therapy is an international, peer-reviewed, open access journal focusing on the pathological basis of all cancers, potential targets for therapy and treatment protocols employed to improve the management of cancer patients. The journal also focuses on the impact of management programs and new therapeutic agents and protocols on

patient perspectives such as quality of life, adherence and satisfaction. The manuscript management system is completely online and includes a very quick and fair peer-review system, which is all easy to use. Visit http://www.dovepress.com/testimonials.php to read real quotes from published authors.

Submit your manuscript here: http://www.dovepress.com/oncotargets-and-therapy-journal 